\title{
Optimization of the Split-Operator Method for Modeling of Quantum System Time Evolution
}

\author{
D. V. Vlasov \\ Physics Faculty, Dept. of Theoretical Physics \\ Herzen State Pedagogical University of Russia \\ St.-Petersburg 191186, Russia
}

Received: April 19, $2005 \quad$ Accepted: August 21, 2005

\begin{abstract}
A continuing and important problem in modeling physical processes deals with creating optimum calculation algorithms. These algorithms should provide the necessary precision yet not demand a lot of storage and time for evaluations. In the present paper the numerical integration of the non-stationary Schrödinger equation (NSSE) is considered. There are many numerical methods of integration of this equation to the present moment. In this paper, we explore the split-operator method (SOM) and consider a way of lessening time computing expenses in view of specificity of the given method. We also consider the choice of optimum discretization steps in coordinate and momentum space. The computer application that we outline is applicable, in method and preparation, to the solution more difficult problems, and it has been applied to simple chemical reactions. We use standard atomic units to simplify equations in the analysis that follows.
\end{abstract}

\section{STATEMENT OF THE PROBLEM}

Integration of the non-stationary Schrödinger equation begins with the definition of a state vector $\left|\Psi\left(t_{0}\right)\right\rangle$ that represents a system at an initial moment of time $t_{0}$. The state vector at any other moment of time $t$ is obtained as the result of the evolution operator of system (the propagator) on this state vector $\left|\Psi\left(t_{0}\right)\right\rangle$.

This operator follows from the formal solution of the NSSE [1],

$$
\begin{gathered}
i \frac{\partial}{\partial t}|\Psi(t)\rangle=\hat{H}|\Psi(t)\rangle \\
|\Psi(t)\rangle=\hat{U}\left(t, t_{0}\right)\left|\Psi\left(t_{0}\right)\right\rangle
\end{gathered}
$$

where the operator

$$
\hat{U}\left(t, t_{0}\right)=e^{-i\left(t-t_{0}\right) H}
$$

is a propagator of the system whose activity should be interpreted as action of the respective operational exponential power series. The form of Hamiltonian $H$ depends on the particular state vector representation used. It is natural to use momentum and coordinate representations since the kinetic energy operator and potential operator that make a Hamiltonian will be local in these representations.

Thus, at each given moment of time it is necessary to set functions

and

$$
\Psi(\vec{x}, t)=\langle\vec{x} \mid \Psi(t)\rangle
$$

$$
\Psi(\vec{k}, t)=\langle\vec{k} \mid \Psi(t)\rangle .
$$

Transformation between these two representations is carried out using Fourier transforms,

$$
\begin{aligned}
\Psi(\vec{k}, t) & =\int\langle\vec{k} \mid \vec{x}\rangle\langle\vec{x} \mid \Psi(t)\rangle d \vec{x} \\
\Psi(\vec{x}, t) & =\int\langle\vec{x} \mid \vec{k}\rangle\langle\vec{k} \mid \Psi(t)\rangle d \vec{k}
\end{aligned}
$$

Here $\langle\vec{k} \mid \vec{x}\rangle=e^{-i \vec{k} \vec{x}}$, and $\langle\vec{x} \mid \vec{k}\rangle=e^{i \vec{k} \vec{x}}$. The basic problem is that of evaluating the action of the evolution operator on a wave 
function, in coordinate or momentum space, at time $t_{0}$. It is the result of this operation, the wave function that represents a state of system at the moment of time $t$, which interests us.

\section{EVALUATION OF ACTION OF THE PROPAGATOR USING THE SPLIT- OPERATOR METHOD}

The problem mentioned in the previous point cannot be solved analytically for most cases of practical interest. Therefore it is necessary to carry out some numerical calculations. $F$ or their realization, it is necessary to set some time interval $\left[t_{0}, t\right]$ and to break it into big enough number $N_{t}$ of much smaller time steps. This gives us a set of time steps $\left\{t_{l} \mid l=0,1,2, \ldots, N_{t}\right\}$. The length of each small time step is usually determined by first considering the micro process at hand. In comparison with the last time, the first should be small number. Let's put it everywhere the same and denote the time step by $\Delta t$. To each moment $t_{1}$ corresponds to the functions $\Psi\left(\vec{x}, t_{l}\right)$ and $\Psi\left(\vec{k}, t_{l}\right)$.

Let the wave function of system at the initial moment of time $t_{0}$ be known. If operator $\hat{U}\left(t_{0}, t_{0}+\Delta t\right)$ acts on it, a wave function for time $t_{0}+\Delta t$ results. A small value of $\Delta t$ is an important condition. It allows us to apply some approximate computing cycle, whose precision can be made sufficiently good if $\Delta t$ is small enough. By consistently applying this computing cycle at all subsequent moments of time $t_{l}$, it is possible to obtain, ultimately, information on system at time $t$.

Under these conditions, we have applied the split-operator method enunciated in [2]. In doing this, we have used the symmetric decomposition of $\hat{U}\left(t_{l}, t_{l}+\Delta t\right)$, precise up to second order in $\Delta t$,

$$
\hat{U}\left(t_{l}, t_{l}+\Delta t\right)=e^{-i \frac{\hat{T}}{2} \Delta t} e^{-i \hat{V} \Delta t} e^{-i \frac{\hat{T}}{2} \Delta t}+O\left(\Delta t^{3}\right)
$$

If $\hat{T}$ represents the kinetic energy operator and $\hat{V}$ potential energy operator, then it is possible to write,

$$
\left|\Psi\left(t_{l}+\Delta t\right)\right\rangle \approx e^{-i \frac{\hat{T}}{2} \Delta t} e^{-i \hat{V} \Delta t} e^{-i \frac{\hat{T}}{2} \Delta t}\left|\Psi\left(t_{l}\right)\right\rangle
$$

A consecutive operation of three exponents in (4) on the state vector of system at present time gives the state vector that approximately characterizes system in the following $\Delta t$ moment of time.

Each exponential operator contains only $T$ or $V$. This decomposition of the evolution operator is convenient due to the following reasons. As mentioned above, the kinetic energy operator is local in the momentum representation and similarly for the potential energy operator in coordinate representation. If the state vector is represented first by function $\Psi\left(\vec{k}, t_{l}\right)$, then the acting on it with operator $e^{-i \frac{\hat{T}}{2} \Delta t}$ reduces to common multiplication with the respective complex exponent,

$$
\begin{aligned}
& \Psi_{T / 2}^{(1)}\left(\vec{k}, t_{l}\right)=e^{-i \frac{\hat{T}}{2} \Delta t} \Psi\left(\vec{k}, t_{l}\right)= \\
& =e^{-i \frac{k^{2}}{4 m} \Delta t} \times \Psi\left(\vec{k}, t_{l}\right)
\end{aligned}
$$

Then it is possible to subject the function obtained as a result of this operation to Fourier transform (3b) and to find its image $\Psi_{T / 2}^{(1)}\left(\vec{x}, t_{l}\right)$ in coordinate space.

Analogously, the obtained result is multiplied by the operator

$$
\begin{gathered}
e^{-i V(\vec{x}) \Delta t}, \\
\Psi_{V}^{(2)}\left(\vec{x}, t_{l}\right)=e^{-i V(\vec{x}) \Delta t} \times \Psi_{T / 2}^{(1)}\left(\vec{x}, t_{l}\right)
\end{gathered}
$$

At last, let's obtain Fourier transform (3a) and multiply the result by a complex exponent in the right part of (6). Doing this we have,

$$
\begin{aligned}
& \Psi\left(\vec{k}, t_{l+1}\right)=\Psi\left(\vec{k}, t_{l}+\Delta t\right) \\
& =\Psi_{T / 2}^{(3)}\left(\vec{k}, t_{l}\right)=e^{-i \frac{k^{2}}{4 m} \Delta t} \times \Psi_{V}^{(2)}\left(\vec{k}, t_{l}\right)
\end{aligned}
$$

The operations described above allow us to carry them out one step at a time for the given interval $\left[t_{0}, t\right]$. It is necessary to make $N_{t}$ of such steps. Each subsequent step 
uses the result of the previous time step. After $N_{t}$ iterations, we obtain a wave function that approximately corresponds to the action of propagator $\hat{U}\left(t_{0}, t\right)$ on function $\Psi\left(\vec{k}, t_{0}\right)$.

\section{USING A DISCRETE FOURIER TRANSFORM FOR A PROBLEM OF EVOLUTION OF A WAVE PACKET AND ITS DISADVANTAGE}

To embody on a computer the described computing cycle it is necessary to present functions as tables. As we deal with separate values of continuous functions, it is necessary to apply discrete Fourier transforms on each time step. This can be written down in the following way for the one-dimensional case,

$$
\begin{aligned}
& \Psi\left(k_{n}, t_{l}\right)=\frac{1}{\sqrt{2 \pi}} \sum_{m=0}^{N-1} e^{-i k_{n} x_{m}} \Psi\left(x_{m}, t_{l}\right) \Delta x \\
& \Psi\left(x_{n}, t_{l}\right)=\frac{1}{\sqrt{2 \pi}} \sum_{m=0}^{N-1} e^{i k_{m} x_{n}} \Psi\left(k_{m}, t_{l}\right) \Delta k
\end{aligned}
$$

where $\left\{x_{n} \mid n=\overline{0,(N-1)}\right\}$ is a set of $\mathrm{x}$ coordinate values, the $\left\{k_{n} \mid n=\overline{0,(N-1)}\right\}$ are values of momentum $k$, and $\Delta x$ is the distance between adjacent coordinate values, with $\Delta k$ a step in the momentum variable. Usually, there is no sense in making the last two quantities depend on the index. It matters only if we are interested in the behavior of the function in some subinterval and want to diminish the step of discretization. But then it is easier to allocate this subinterval from the remaining interval and to reallocate the discrete steps. Thus, certainly, it is necessary to take care of a correct lacing on boundaries.

Let's consider closely the sum in (9). Replacing $k_{n}$ for the continuous variable, it is possible to show that the function obtained is periodic because of the periodicity of complex exponents in the sum. Really, one can write down following relations,

$$
e^{-i k x_{m}}=e^{-i k \Delta x \cdot m-i 2 \pi \cdot m}=e^{-i m \Delta x\left(k+\frac{2 \pi}{\Delta x}\right)}
$$

$$
e^{-i k_{n} x_{m}}=e^{-i(\Delta k \Delta x) \cdot n m-i 2 \pi \cdot m}=e^{-i \frac{2 \pi}{N}(n+N) \cdot m}
$$

Inconvenience in the immediate application of Fourier transforms in practice can be exhibited for a small value of $\Delta x$. If the quantity $x_{\max }$ is constant then diminution of $\Delta x$ by some number of times gives the same increase in $k_{\max }$. Thus, $\Delta k$ remains the same. The domain for which $\Psi\left(k, t_{l}\right)$ is nonzero also doesn't vary. As a result, diminution of a discrete step size in coordinate space implies the growth of number of useless zero values of function. These ones correspond to the area of great values of wave numbers. The probability of detection of system is actually equal to zero in this place.

The situation worsens considerably if the derivative of the potential becomes large or infinite (a vertical wall for example) within the coordinate space of interest. The true function $\Psi\left(k, t_{l}\right)$ is not smooth in this case. Performing direct and inverse discrete Fourier transforms on each time step, one can lose a lot of information about wave packet evolution. Therefore it is desirable to simulate a process using a small step of discretization, both in coordinate and momentum space. Certainly, it is necessary to use some fast algorithm of discrete Fourier transform (FFT).

\section{CORRECT CHOICE OF STEPS IN COORDINATE AND MOMENTUM SPACE}

The above mentioned problem includes two components. Let's denote by $M_{1}$ the number of nonzero values of $\Psi\left(k_{n}, t_{l}\right)$. After completion of a fast Fourier transform, the number of ones is equal $M_{2}$. In the general case $M_{1}$ is not equal to $M_{2}$. Consequently, it is necessary to obtain the same numbers of nonzero values of functions in both spaces. Then the amount of information of system is balanced in both representations. The second problem lies in choosing the quantity of nonzero values that will provide a small interval of discretization. Let's consider a Gaussian wave packet for example. We must equate numbers of 
points that correspond to the localization areas of the wave packet in momentum and coordinate space,

$$
\frac{\sigma_{x}}{\Delta x}=\frac{\sigma_{k}}{\Delta k}
$$

Quantities $\sigma_{x}$ and $\sigma_{k}$ are the typical breadths of wave packets. Putting $\sigma_{k}=1 /\left(2 \sigma_{x}\right)$ and taking into account expressions for $\Delta x$ and $\Delta k$, we obtain the following result,

$$
\frac{x_{\max }^{2}}{\sigma_{x}^{2}}=4 \pi N
$$

This relation ensures that the solution will correctly assign wave function values on a discrete grid. It is equivalent to equation,

$$
x_{\max } \sigma_{k}=\sqrt{\pi N}
$$

If there are no restrictions on values of $x_{\max }$ then the assignment of the rational step in momentum space can be realized by selecting the quantity of the former and the amount of subintervals $N$. The choice of values of these quantities depends on parameters of a specific problem. The main parameters, which define the mentioned choices, are the typical radius of action of the potential, the average kinetic energy of system and time of duration of the process.

If quantity of an interval $x_{\max }$ cannot be changed in the arbitrary limits it is possible to resort to a simple alternate method. From the original set of values of the function $\Psi\left(x_{n}, t_{l}\right)$ that correspond to quantities $\left\{x_{n} \mid n=\overline{0,(N-1)}\right\}$, some of them are excluded. For example, if the standard algorithm with number of points $N$ $=2^{p}$ for execution of the FFT is used, the exclusion of every $N_{D}$-th values from the table of function is carried out. $N_{D}$ is equal to the integer power of two. It corresponds to the increase by $N_{D}$ times of step $\Delta x$. The remaining functional values are put in the relevant one-dimensional array one after another. The number of places in this array remains equal $N$. The free places at the end of it are filled by nulls. It corresponds to the decrease of the interval of wave numbers and a step of discretization in momentum space the respective number of times.
Hence, the number of nonzero values of function $\Psi\left(k_{n}, t_{l}\right)$ increases. The remaining functional values in coordinate space correspond to former values of coordinate $x$. Thus, owing to loss of some redundant information about wave function $\Psi\left(x_{n}, t_{l}\right)$, we have obtained more detailed information about function $\Psi\left(k_{n}, t_{l}\right)$ for each instant $t_{\text {. }}$.

Application of such methods allows us "to balance" the quantities of useful information about a system, when a state vector is associated with functions in two different representations.

\section{USE OF A METHOD "FROM NUMBER TO NUMBER" FOR FAST MULTIPLICATION OF A COMPLEX NUMBER TO A COMPLEX EXPONENT}

Use of a fast Fourier transform in combination with the split-operator method involves numerous multiplications of a complex number to a complex exponent. This operation demands knowledge of the values of trigonometric functions sine and cosine. These values can be obtained from computed tables or computed by power series. Both of these modes can demand considerable time expenditures. Let's designate $C$ as some complex number. Also we denote $\operatorname{Re}(C)=X$ and $\operatorname{Im}(C)=Y$. This can be represented as follows

$$
\left(\begin{array}{l}
X^{(\alpha)} \\
Y^{(\alpha)}
\end{array}\right)=\left(\begin{array}{cc}
\cos (\alpha) & -\sin (\alpha) \\
\sin (\alpha) & \cos (\alpha)
\end{array}\right) \times\left(\begin{array}{c}
X \\
Y
\end{array}\right)
$$

It is equivalent to equality $C^{(\alpha)}=C \times e^{-i \alpha}$.

There is an optimal method of realizing this operation, termed as the method "from number to number" [3]. The name of the method is based by receiving one binary digit on each step of the algorithm.

Let's consider a classical version of this method. An initial two-dimensional vector rotates clockwise and anticlockwise in the $2 \mathrm{D}$ plane through angles $Q_{i}$, defined as follows

$$
\begin{aligned}
& Q_{1}=\pi / 2 \\
& Q_{i}=\arctan \left(2^{-(i-2)}\right), \quad i=2,3,3 \ldots
\end{aligned}
$$




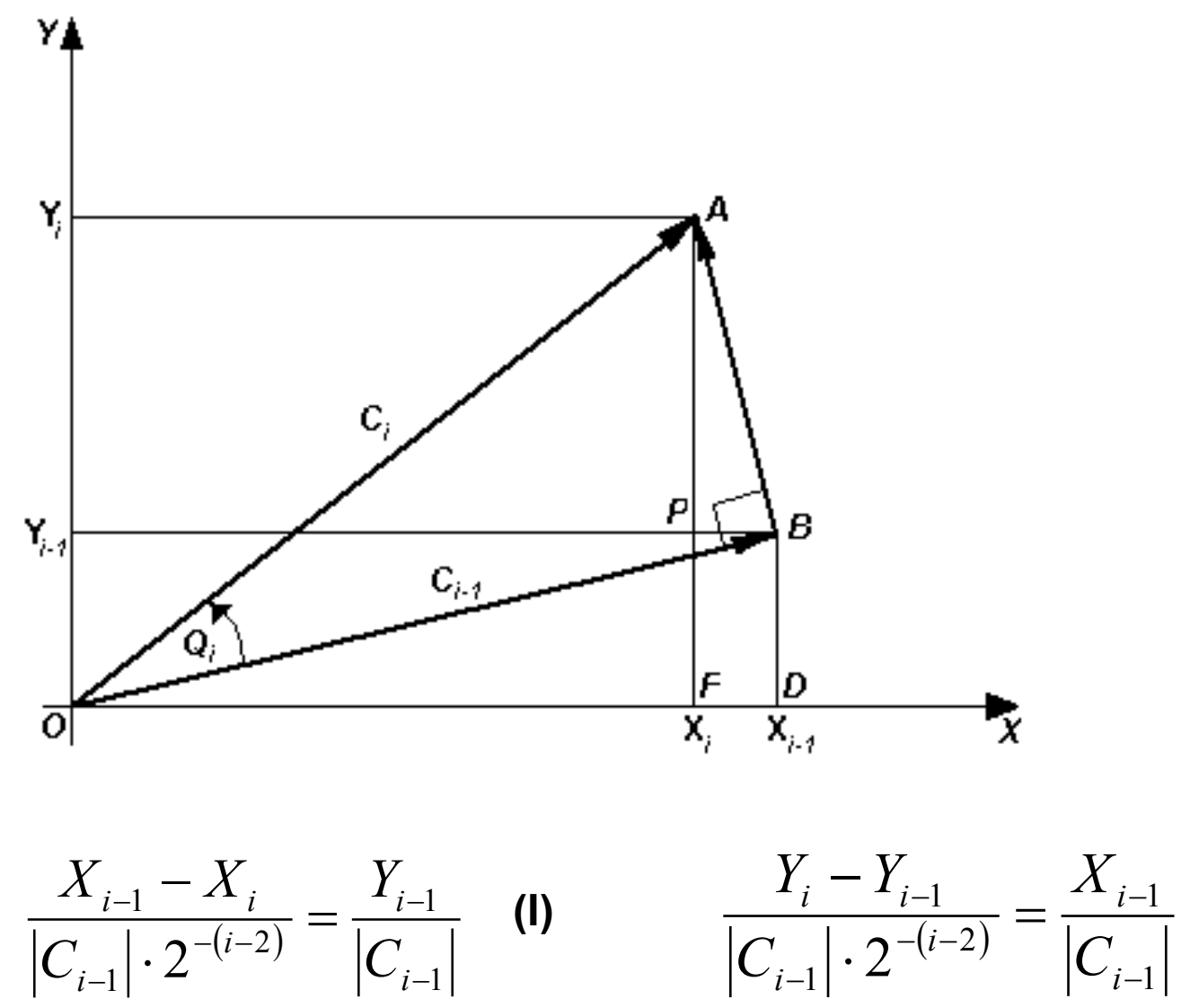

Figure 1. A two-dimensional vector $\mathbf{C}$ rotates in the $2 \mathrm{D}$ plane, a simple classical example of the "from number to number" method of iterating for the final form of a state (in this case, C).

The iterative relations that tell us the final components of this vector, following all rotations, are

$$
\begin{gathered}
X_{1}=-\varepsilon_{1} \cdot Y, \quad Y_{1}=\varepsilon_{1} \cdot X \\
X_{i}=X_{i-1}-\varepsilon_{i} \cdot Y_{i-1} \cdot 2^{-(i-1)} \\
Y_{i}=Y_{i-1}-\varepsilon_{i} \cdot X_{i-1} \cdot 2^{-(i-1)} \\
\alpha_{i}=\alpha_{i-1}-\varepsilon_{i} \cdot Q_{i}
\end{gathered}
$$

Quantities $X_{i}$ and $Y_{i}$ are projections of the vector $C$ onto the $x$-axis and the $y$-axis.

Formulas (17a-c) can be obtained the following way. Let us assume that the $i^{\text {th }}$ rotation is anticlockwise. Look at Figure 1. One can prove easily that the triangles $A B P$ and $O B D$ are similar. Then the equations (I) and (II) are true. It is possible to write down the analogous equations for clockwise rotation. As a result we obtain recursion relations $(17 a-c)$.

Equations (16) determine the first non-typical rotation by angle $\pi / 2$. The initial value of index $i$ still begins from two. Quantity $\varepsilon_{1}$ is equal 1 , if $\alpha>0$ and -1 otherwise. Quantity $\varepsilon_{i}$ is defined by sign of $\alpha_{i-1}$,

$$
\varepsilon_{i}=\operatorname{sgn}\left(\alpha_{i-1}\right)
$$

The number of iterations is determined by the digit capacity used for floating point numbers. If it is equal $n$ then the number of rotations needed is $n / 2+1$.

As is easy to see from Figure 1, the described method has an important deficiency. This is the length magnification 
of the turned vector. Overall lengthening $K$ can be expressed by formula,

$$
K=\prod_{i=2}^{n / 2+1}\left(1+2^{-2(i-2)}\right)^{\frac{1}{2}}
$$

Thus, it is necessary to divide an input or output vector by quantity $K$.

With a view to unifying this algorithm and its speed of execution, it is possible to act differently. The quantity $1 / K$ can be represented by the following expression,

$$
\frac{1}{K}=0.5 \cdot \prod_{j=1}^{9}\left(1 \pm 2^{-\left|P_{j}\right|}\right)
$$

convenient for 32 bit numbers. set Let numbers $P_{j}$ belong to a $P=\{3,4,6,11,-14,-19,22,29,30\}$. They are obtained by selection. Then the neutralization of the above lengthening of vector is carried out with the following formulas:

$$
\begin{gathered}
X_{0}=0.5 \cdot Y_{0}, Y_{0}=0.5 \cdot Y_{0} \\
X_{j}=X_{j-1}-\varepsilon_{j} \cdot Y_{j-1} \cdot 2^{-\left|P_{j}\right|} \\
Y_{j}=Y_{j-1}-\varepsilon_{j} \cdot X_{j-1} \cdot 2^{-\left|P_{j}\right|} \\
\varepsilon_{j}=\operatorname{sgn}\left(P_{j}\right)
\end{gathered}
$$

Index $j$ varies from 1 up to 9 . The number of iterations $n$ equals 32 for the given set $P$.

It is necessary to take care that division into the integer power of two can be carried out with the bit shift on the respective number of binary positions. This operation is also executed quickly as a command of addition. It is necessary to note that the method "from number to number" is not effective when it is used without the bit shift. Time of calculation can increase more than two times in comparison with the usual methods of evaluations mentioned in the beginning of this point.

Calculations under formulas (17) and (20) can be executed in a parallel way on a three-processor computer. One operation of rotation is equivalent to one command of multiplication in this case. Calculation on one processor is equivalent to three commands of multiplication, accordingly.

\section{THE INFERENCE}

The combination of the method 'from number to number' and the algorithm of the fast Fourier transform allow achieving high performance on time. We infer that it is especially effective when used along with the split-operator method for simulation of time evolution of a quantum micro system.

Numerical calculations were carried out by author for collisions $\mathrm{H}+\mathrm{H}$ and $\mathrm{H}+\mathrm{H}_{2}$ on a one-processor computer. The value of time expenses decreased by up to $80 \%$ from the usual one employed, depending on parameters of calculation.

Modern computer simulations of quantum systems can be very laborious. They may take a lot of time (from several hours up to several days or more). A definite improvement of time efficiency is a significant outcome.

\section{REFERENCES}

1. See any intermediate quantum mechanics textbook, for example, J.J. Sakurai, Modern Quantum Mechanics (Addison-Wesley Publishing Co., Reading, Massachusetts, USA, 1995), chapter 2.

2. N.Balakrishnan, C.Kalyanaraman, N.Sathyamurthy, "Time-dependent quantum mechanical approach to reactive scattering and related processes," Physics Reports 280 (1997) pp. 79-144.

3. Z.S.Kuzin, "Ultrafast algorithm for evaluation of Fourier transform," Wissenschaftliche Beiträge, Heft 4/81 (1981) pp. 22-26 [in Russian].

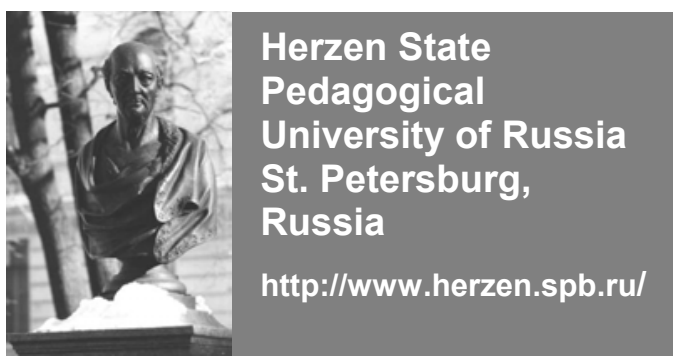

